\title{
Primate phylogenomics: developing numerous nuclear non-coding, non-repetitive markers for ecological and phylogenetic applications and analysis of evolutionary rate variation
} Zuogang Peng1, Navin Elango ${ }^{1}$, Derek E Wildman ${ }^{2}$ and Soojin V Yi*1

\author{
Address: ${ }^{1}$ School of Biology, Institute of Bioscience and Bioengineering, Institute of Biosystems, Georgia Institute of Technology, Atlanta, GA \\ 30332, USA and 2Center for Molecular Medicine and Genetics, Wayne State University School of Medicine, Detroit, MI 48201, USA \\ Email: Zuogang Peng - zpeng6@mail.gatech.edu; Navin Elango - navin@gatech.edu; Derek E Wildman - dwildman@med.wayne.edu; \\ Soojin V Yi* - soojinyi@gatech.edu \\ * Corresponding author
}

Published: 26 May 2009

BMC Genomics 2009, 10:247 doi:10.1 186/|47|-2/64-10-247
Received: 5 November 2008

Accepted: 26 May 2009

This article is available from: http://www.biomedcentral.com/I47I-2/64/I0/247

(c) 2009 Peng et al; licensee BioMed Central Ltd.

This is an Open Access article distributed under the terms of the Creative Commons Attribution License (http://creativecommons.org/licenses/by/2.0), which permits unrestricted use, distribution, and reproduction in any medium, provided the original work is properly cited.

\begin{abstract}
Background: Genetic analyses are often limited by the availability of appropriate molecular markers. Markers from neutrally evolving genomic regions may be particularly useful for inferring evolutionary histories because they escape the constraints of natural selection. For the majority of taxa however, obtaining such markers is challenging. Advances in genomics have the potential to alleviate the shortage of neutral markers. Here we present a method to develop numerous markers from putatively neutral regions of primate genomes.
\end{abstract}

Results: We began with the available whole genome sequences of human, chimpanzee and macaque. Using computational methods, we identified a total of 280 potential amplicons from putatively neutral, non-coding, non-repetitive regions of these genomes. Subsequently we amplified, using experimental methods, many of these amplicons from diverse primate taxa, including a ringtailed lemur, which is distantly related to the genomic resources. Using a subset of 10 markers, we demonstrate the utility of the developed markers in phylogenetic and evolutionary rate analyses. Particularly, we uncovered substantial evolutionary rate variation among lineages, some of which are previously not reported.

Conclusion: We successfully developed numerous markers from putatively neutral regions of primate genomes using a strategy combining computational and experimental methods. Applying these markers to phylogenetic and evolutionary rate variation analyses exemplifies the utility of these markers. Diverse ecological and evolutionary analyses will benefit from these markers. Importantly, methods similar to those presented here can be applied to other taxa in the near future.

\section{Background}

The accumulating body of draft genome assemblies from diverse animal species offers unprecedented opportunities for resolving the tree of life. A key component of empirical studies of molecular evolutionary phenomena is the analysis of molecular markers. To date, most molecular phylogenetic studies have relied on sequences from less than a few dozen genes. Mitochondrial DNA sequences have 
been the workhouse of phylogenetic and phylogeographic studies for the past two decades (e.g. $[1,2])$. DNA barcoding, a technique is increasingly used to identify species, is reliant on mtDNA [3]. While these methods have strengths, each carries some implicit limitations. First, because mtDNA markers are maternally inherited, the ability to infer evolutionary events from the perspective of both sexes is limited. In addition, the reduced effective population size of mtDNA compared to that of nuclear markers could confound population genetic inferences. Moreover, it is now well established that mtDNA sequences are often incorporated into nuclear genomes in diverse taxa, including humans and other primates $[4,5]$.

Markers from single-copy nuclear DNA are free from the aforementioned problems. Often used single-copy nuclear DNA markers include conserved exons and genes. However, the effects of natural selection on these markers can result in homoplasy that has the potential to mislead phylogenetic analyses [6]. Similarly, genes that experienced positive selection in specific lineages (e.g., RNases evolution in leaf monkeys, [7]) may have inaccurate phylogenetic signals (i.e., they suffer long branch attraction due to increased number of nonsynonymous substitutions in specific lineages). Conversely, genes that have a history of strong purifying selection may harbor few phylogenetically informative sites, which make them unsuitable for population genetic studies or phylogenetic resolution in rapidly evolving taxa.

In addition to sequence based markers, events such as the insertion of transposable elements into ancient genomes provide excellent phylogenetic information [8]; yet these markers provides little information on rates of nucleotide substitution. Because of these limitations, neutrally evolving nuclear DNA sequence markers may provide the best source of data for phylogenetic inference and estimates of evolutionary rate variation.

Advances in genomics give molecular evolutionary studies an extraordinary opportunity to establish numerous nuclear, putatively neutral molecular markers. Genomes of many taxa, including those of primates, have a large amount of non-coding DNA, which can be used to infer genomic divergence and the influence of neutral mutation rate variation [911]. Therefore, we can obtain large numbers of putatively nuclear molecular markers from non-coding regions. Even though currently most taxa lack genome scale information, sequencing technologies are rapidly improving, and it will become progressively easier to obtain genome sequences. The challenges then are, to utilize genomic information to develop markers that can be used in a variety of ecological, phylogenetic, and evolutionary applications.

Here we present a method for developing and utilizing numerous non-coding, non-repetitive markers in pri- mates. The availability of whole-genome sequences of primates combined with their well-resolved phylogenetic relationships makes them an excellent model system in which to devise computational and experimental tools to search for useful molecular markers. Moreover, such markers from primate genomes are potentially useful because they could be applied to the several outstanding phylogenetic problems in primates (for example, [1215]). Such molecular markers also could serve as a resource for understanding the genetic history of primate populations, a topic of study of interest to molecular ecologists, primate biologists, and anthropologists.

We demonstrate the utility of these markers by applying them to phylogenetic and evolutionary analyses. We first constructed a sample data set of ten non-coding, nonrepetitive markers from ten diverse primate taxa, including a strepsirrhine species rather distantly related to existing genomic resources. We reconstructed the correct species phylogeny with high confidence and uncovered significant evolutionary rate variation between lineages. Furthermore, we have uncovered new and statistically significant rate variation between some primate lineages. Thus, our markers may contribute understanding the patterns and causes of neutral evolutionary rate variation between lineages. We propose that the methods outlined here can be used in diverse taxa to address phylogenomic and population genetic questions.

\section{Results}

\section{Amplification of potential markers}

Our preliminary screen for orthologous non-repetitive and non-coding segments based on a three-way alignment the genomes of two hominid apes (human, Homo sapiens; and common chimpanzee, Pan troglodytes) and the rhesus macaque (Macaca mulatta, an Old World monkey) resulted in nearly 10,000 candidate amplicons. Subsequently, we designed 280 (212 from autosomes and 68 from the $\mathrm{X}$ chromosome) primer pairs in regions ranging from $\sim 300$ to $\sim 1200$ base pairs long, with an average length of $\sim 600$ bps.

We then used the polymerase chain reaction (PCR) to amplify these markers from the following primate species: gorilla (Gorilla gorilla, a nonhuman hominid), anubis baboon (Papio anubis, an Old World monkey), spider monkey (Ateles geoffroyi, a New World monkey) and tamarin (Saguinus labiatus, a New World monkey), and ringtailed lemur (Lemur catta, a prosimian). Two hundred primer pairs ( $70 \%)$ amplified a single band from $G$. gorilla, 170 primer pairs ( $60 \%)$ amplified a single band for P. anubis, $116(\sim 41 \%)$ and 106 ( 38\%) primer pairs amplified a single band for A. geoffroyi and S. labiatus respectively. Furthermore, we amplified single bands in 18 markers $(\sim 6 \%)$, from a phylogenetically distant clade from the genome resources, $L$. catta. Sequences, locations 
in the human genome, and the applicable range of phylogeny of these markers (total 280 non-coding, non-repetitive markers) are listed the Additional file 1 [see Additional file 1].

We chose 10 markers (6 autosomal and 4 X-linked) for subsequent analyses (Table 1 ). These markers are likely to be orthologous, for the following reasons. First, these ten markers are unique genomic regions in the human genome (i.e. they are not included in segmentally duplicated regions, nor do they have closely related paralogous sequences in the genome, as determined by homology searches). Second, primers for all the ten markers produced single bands in PCR reactions in the primate species we tested. Third, when we used the experimentally amplified sequences as a query to search other genomes using BLAT or BLAST programs, all of these produced a single hit in the expected locations. Fourth, genetic divergences of these markers also fall within the expected ranges of neutrally evolving orthologous markers (see below). However, we caution that it is possible that these markers are not orthologous, given that most primate genomes are far from complete, and that unknown copy number variation may exist between and within species. Thus, whether the amplified markers of these regions from other primate genomes truly represent orthologous segments can only be determined by comparing finished, high-fold coverage, whole genome sequences from multiple individuals of the primate species in our study. The markers amplified and sequenced in this study are deposited in the GenBank (accession numbers GQ175181GQ175229).

We also retrieved these 10 regions from the publicly available genome sequences of the following five species: human (H. sapiens), common chimpanzee (P. troglodytes), orangutan (Pongo pygmaeus abelii), rhesus macaque $(M$. mulatta), and the common marmoset (Callithrix jacchus). All sequences, except for the $\mathrm{X} 1$ marker from the chimpanzee and the A5 marker from macaque, were of high quality (meaning, $\leq 2$ ambiguous sites). The chimpanzee
$\mathrm{X} 1$ and the macaque $\mathrm{A} 5$ marker had a substantial number of ambiguous sites, suggesting that the two particular markers may potentially include sequencing and/or assembly errors. Therefore, we experimentally amplified the two regions from the corresponding species and used them in further analyses.

Subsequent analyses were all based on these 10 markers. We used the GBlocks program [8] to remove poorly aligned sites. The alignment information and nucleotide content of the markers are shown in Table 2. A1 had the longest alignment with 778 aligned sites. The GC content of the 10 markers ranged from $30.5 \%$ in $\mathrm{A} 3$ to $54.3 \%$ in A2, with an average of $38.7 \%$ for the concatenated dataset. We tested whether base frequencies across taxa are homogenous using a chi-square test for each of the 10 markers and found no sign of heterogeneity among taxa ( $P>0.1$ for all markers, results not shown).

\section{Genetic divergence}

To estimate the genomic divergence for these newly determined markers, we calculated the pairwise Kimura twoparameter distances [16] among the 10 primate species. A subset of pairwise divergences is shown in Table 3. All pairwise distances for the species compared here are shown in the Additional file 2 [see Additional file 2].

There is a substantial variation of evolutionary distances among markers. For example, the distance between human and chimpanzee ranges from $0.59 \%$ in $\mathrm{A} 2$ to $2.34 \%$ in X2. Such regional heterogeneity has been observed before and could be caused by several different factors, such as the difference in the proportion of sites susceptible to mutations caused by DNA methylation $[17,18]$. Nevertheless, this variation is within the range of $0 \%-2.91 \%$ observed in previous studies using non-repetitive and non-coding regions $[9,19]$. The mean humanchimpanzee divergence from these 10 segments is $1.53 \%$ $\pm 0.16 \%$, which is close to previous estimates $(1.24 \% \pm$ $0.07 \%$ [9], and $1.19 \% \pm 0.02 \%$ [19]). Thus, non-coding, non-repetitive markers developed in this study exhibit

Table I: The ten non-repetitive and non-coding (intron or intergenic regions) markers amplified and analyzed in this study.

\begin{tabular}{cccc}
\hline Marker & Corresponding human chromosome & ENCODE region & hg/8 location \\
\hline A1 & 7 & Yes & $116019952-116020821$ \\
A2 & 10 & No & $130647230-130647739$ \\
A3 & 13 & No & $91672658-91673369$ \\
A4 & 15 & No & $84502360-84503129$ \\
A5 & 16 & Yes & $26155304-26155905$ \\
A6 & 21 & Yes & $34098146-34098878$ \\
X1 & $X$ & No & $29000135-29000689$ \\
X2 & $X$ & No & $31344540-31345210$ \\
X3 & $X$ & No & $97879736-97880312$ \\
X4 & $X$ & No & $141551659-141552259$
\end{tabular}


Table 2: Characteristics of the 10 non-repetitive and non-coding segments and for concatenated dataset.

\begin{tabular}{|c|c|c|c|c|c|c|c|c|}
\hline \multirow[b]{2}{*}{ Locus } & \multirow[b]{2}{*}{$\begin{array}{c}\text { \# aligned sites } \\
\text { (\# analyzed)। }\end{array}$} & \multirow[b]{2}{*}{ \# variable Sites } & \multirow[b]{2}{*}{$\#$ \#I sites ${ }^{2}$} & \multicolumn{4}{|c|}{ Mean base frequencies (\%) } & \multirow[b]{2}{*}{ Model ${ }^{3}$} \\
\hline & & & & A & C & G & $\mathrm{T}$ & \\
\hline Al & $806(778)$ & 271 & 96 & 32.1 & 23.5 & 19.5 & 25.0 & $\mathrm{GTR}+\mathrm{G}$ \\
\hline $\mathrm{A} 2$ & $508(508)$ & 75 & 27 & 22.1 & 23.9 & 30.4 & 23.5 & $\mathrm{HKY}+\mathrm{G}$ \\
\hline A3 & $485(473)$ & 151 & 63 & 31.4 & 15.6 & 14.9 & 38.2 & $\mathrm{GTR}+\mathrm{G}$ \\
\hline A4 & $806(673)$ & 234 & 70 & 28.1 & 21.0 & 21.9 & 28.9 & GTR \\
\hline A5 & $619(569)$ & 205 & 83 & 32.9 & 18.0 & 16.3 & 32.8 & GTR \\
\hline A6 & 631 (569) & 234 & 92 & 34.3 & 14.9 & 17.9 & 32.9 & GTR+I \\
\hline$X I$ & 508 (487) & 145 & 63 & 28.5 & 18.0 & 18.6 & 34.9 & $\mathrm{GTR}+\mathrm{G}$ \\
\hline$\times 2$ & $586(565)$ & 154 & 67 & 30.3 & 18.7 & 20.6 & 30.5 & $\mathrm{GTR}+\mathrm{G}$ \\
\hline$\times 3$ & 466 (449) & 125 & 43 & 33.5 & 17.0 & 17.1 & 32.4 & $\mathrm{GTR}+\mathrm{G}$ \\
\hline$\times 4$ & $528(522)$ & 178 & 79 & 32.8 & 19.9 & 15.2 & 32.1 & GTR+I \\
\hline Concatenated & 6535 (5592) & $179 \mid$ & 684 & 30.6 & 19.3 & 19.4 & 30.7 & $\mathrm{GTR}+\mathrm{G}$ \\
\hline
\end{tabular}

\footnotetext{
I The number of analyzed sites are after removing poorly aligned sites using the Gblocks program [46].

$2 \mathrm{PI}$ sites = parsimony informative sites

${ }^{3}$ Best fitting substitution models determined using the AIC (see text). (GTR = General time reversible model; HKY = Hasegawa-Kishono-Yano model; $\mathrm{G}=$ The shape parameter of a gamma distribution; I = The proportion of invariable sites in the alignment)
}

genetic divergences similar to those obtained from larger scale analyses.

We report that the divergence between baboon and macaque is $1.29 \% \pm 0.16 \%$ for the concatenated dataset (range between $0.39 \%-1.98 \%$ ). This value is similar to an earlier estimate $(1.24 \%$ in [17]). Within the New World monkeys, marmoset and tamarin are more closely related to each other (average distance: $4.02 \% \pm 0.27 \%$ ) than either is to the spider monkey (Table 3 ). This observation is concordant with the generally accepted understanding of platyrrhine phylogeny [14].

We also estimated genetic distances between different primate groups, defined as the mean of all pairwise distances between two groups in a inter-group comparison [20]. The average divergences between apes and Old World monkeys, apes and New World monkeys, and Old World monkeys and New World monkeys are 6.92\% $\pm 0.34 \%$, $10.90 \% \pm 0.39 \%$, and $11.80 \% \pm 0.47 \%$, respectively. Notably, genetic divergences between the spider monkey and the other two New World monkeys (spider monkey versus marmoset [ $5.85 \% \pm 0.35 \%]$; spider monkey versus tamarin $[5.35 \% \pm 0.32 \%]$ ) are comparable to the divergence between apes and Old World monkeys (Table 3 ).

Our data also provide a chance to examine the genetic divergence between ring-tailed lemur and other primate groups based upon nuclear, non-coding, non-repetitive loci. Hominid apes, Old World monkeys and New World

Table 3: Pairwise divergences between some primate species and groups. The Kimura two-parameter model [16] was used to estimate the divergence.

\begin{tabular}{|c|c|c|c|c|c|c|c|c|c|c|c|}
\hline \multirow[b]{2}{*}{ Marker } & \multicolumn{11}{|c|}{ Kimura two-parameter Distance $\left(\times 10^{-2}\right)$} \\
\hline & $\mathrm{H}-\mathrm{C}$ & $\mathrm{H}-\mathrm{O}$ & $\mathrm{H}-\mathrm{MC}$ & $\mathrm{H}-\mathrm{BA}$ & $\mathrm{H}-\mathrm{MR}$ & $\mathrm{H}-\mathrm{SM}$ & H-TA & H-LM & HOM-OWM & HOM-NWM & OWM-NWM \\
\hline Al & 1.30 & 5.08 & 8.63 & 7.18 & 12.11 & 11.77 & 10.64 & 22.31 & 8.27 & 11.79 & 11.93 \\
\hline $\mathrm{A} 2$ & 0.59 & 2.00 & 2.32 & 2.11 & 4.91 & 4.06 & 5.12 & 9.31 & 2.35 & 4.78 & 4.33 \\
\hline A3 & 1.74 & 0.86 & 6.32 & 6.09 & 13.82 & 12.31 & 13.74 & 22.73 & 6.57 & 13.14 & 13.70 \\
\hline A4 & 1.96 & 1.97 & 6.82 & 6.14 & 10.80 & 10.23 & 10.39 & 24.50 & 6.83 & 10.94 & 9.60 \\
\hline A5 & 0.72 & 3.29 & 5.98 & 5.78 & 15.30 & 13.48 & 12.82 & 29.08 & 5.77 & 13.91 & 13.54 \\
\hline A6 & 2.16 & 4.04 & 8.28 & 8.69 & 17.39 & 15.18 & 15.00 & 34.78 & 7.36 & 14.94 & 16.50 \\
\hline XI & 0.62 & 3.37 & 9.54 & 10.24 & 8.87 & 8.38 & 11.49 & 18.73 & 9.59 & 9.76 & 12.66 \\
\hline$\times 2$ & 2.34 & 3.64 & 7.25 & 6.66 & 11.24 & 9.97 & 10.55 & $17.7 \mid$ & 6.40 & 10.38 & 10.97 \\
\hline$\times 3$ & 1.81 & 2.27 & 7.01 & 7.51 & 6.82 & 8.03 & 7.09 & 18.97 & 6.84 & 7.57 & 10.95 \\
\hline$\times 4$ & 2.16 & 5.02 & 9.33 & 8.89 & 10.44 & 11.91 & 12.55 & 21.86 & 8.39 & 11.83 & 14.28 \\
\hline Concatenated & 1.53 & 3.24 & 7.29 & 6.88 & 11.22 & 10.60 & 10.81 & 22.07 & 6.92 & 10.90 & 11.80 \\
\hline
\end{tabular}

Note. $\mathrm{C}=$ chimpanzee, $\mathrm{H}=$ human, $\mathrm{G}=$ gorilla, $\mathrm{O}=$ orangutan, $\mathrm{BA}=$ baboon, $\mathrm{MC}=$ macaque, $\mathrm{MR}=$ marmoset, $\mathrm{SM}=$ spider monkey, $\mathrm{TA}=$ tamarin, $\mathrm{LM}=$ lemur, HOM = hominoids, NWM = New World monkey, OWM = Old World monkey. 
monkeys are on average $22.29 \% \pm 0.75 \%, 23.66 \% \pm$ $0.89 \%$ and $23.45 \% \pm 0.74 \%$ divergent from the ring-tailed lemur based on our data. Previous studies suggested approximately 20-21\% divergence between human and the ring-tailed lemur $[12,21]$. Our estimate of the genetic distance between hominids and the ring-tailed lemur is similar to this value, and these findings also show that Old World monkeys and New World monkeys have diverged slightly more from lemur than have the apes.

\section{Phylogenetic analysis}

We performed phylogenetic analyses using the new markers generated in this study. We used Maximum Parsimony (MP), Neighbor Joining (NJ), and Bayesian Inference (BI) methods to construct phylogenetic trees for each of the 10 markers. The best-fitting substitution model for each marker for Bayesian Inference (BI) was determined by the Akaike information criterion (AIC) as implemented in ModelTest3.7 [22] and MrModelTest2.3 [23] (listed in Table 2). We also performed phylogenetic analyses using the three methods and a maximum likelihood (ML) method of the concatenated data set.
Overall, none of these methods strongly supported conflicting topologies for any single marker. However, individual-marker analyses were less well resolved than the tree based on the concatenated data (compare Figure 1 with Figure 2). Figure 2 represents the total evidence tree obtained from MP, NJ, ML, and $\mathrm{BI}$ of the concatenated dataset. All the methods recovered identical topology with $100 \%$ bootstrap value or 1.0 Bayesian posterior probability support for all clades, except for node 1 (Figure 2). This tree agrees with the current understanding of the primate species tree in most aspect.

The behavior of combining characters from different partitions was evaluated by examining the relative contribution, or utility, of data partitions to resolving relationships of the combined data set. Specifically, partitioned Bremer support (PBS) was calculated using TreeRot3 [24] using the method of Baker and DeSalle [25] to measure the relative contribution of each marker to node support. The larger the partitioned Bremer support is for a given partition at a particular node, the greater the relative contribution of that partition to the support of that node. The sum of all partition lengths for any given node will always
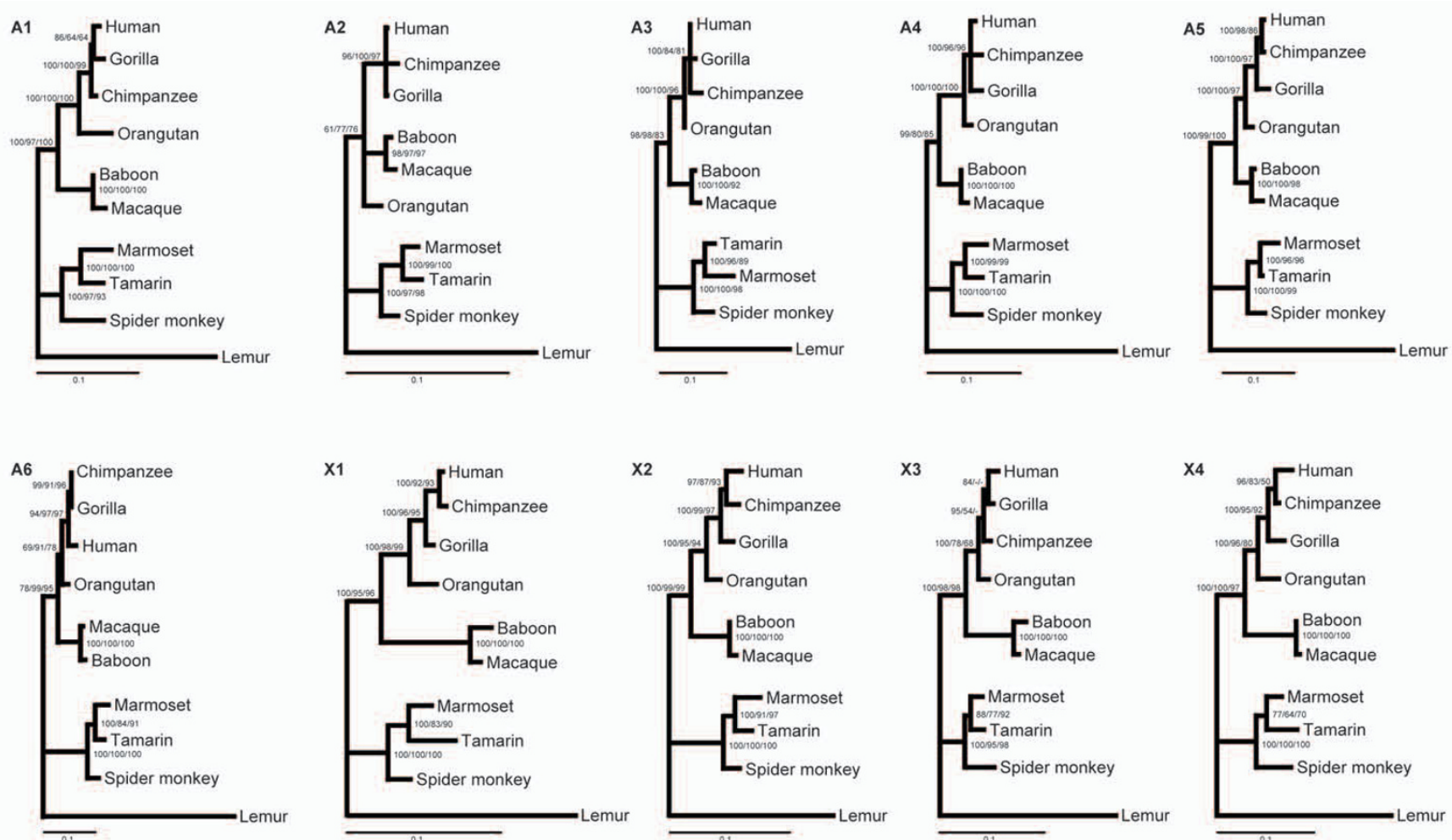

Figure I

Phylogenetic trees for each marker. The numbers at each node were the support values based on Bayesian inference (BI)/ maximum parsimony (MP)/neighbor-joining (NJ) analyses, respectively. The support values $<50 \%$ were indicated using short dash "-" as shown in the figure. 


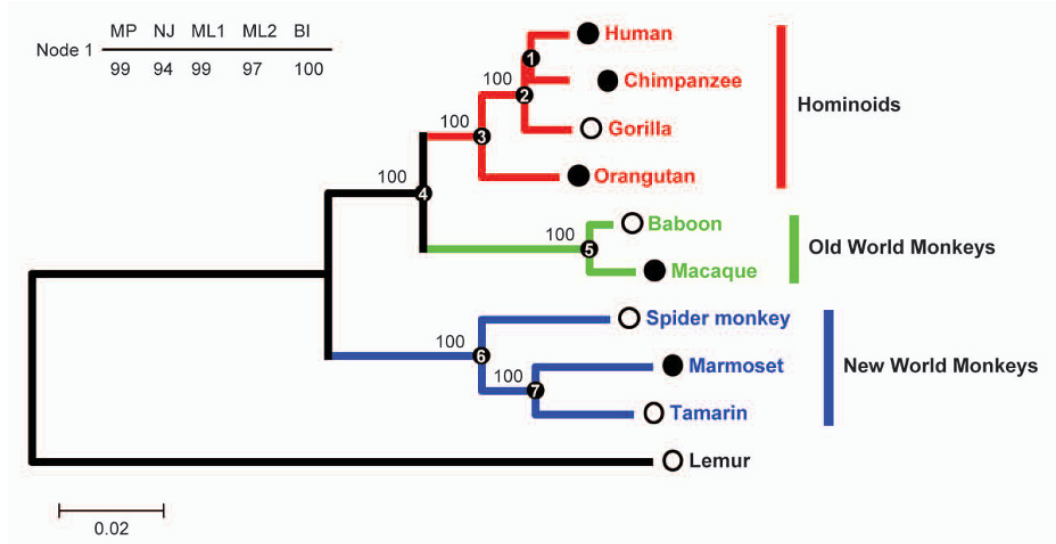

Figure 2

Phylogeny of the ten primates. The tree is derived from the analyses of the concatenated dataset (5592 bp) based on maximum parsimony (MP), neighbor-joining (NJ), maximum likelihood (using Garli [MLI] and PHYML [ML2]), and Bayesian inference $(\mathrm{BI})$. All the nodes (except node I, which indicated otherwise) received $100 \%$ bootstrap proportion and I.0 Bayesian posterior probability support. Branch lengths were optimized using NJ based on Kimura 2-parameter distance model. The solid circle before the taxon name means the data for this taxon were retrieved from UCSC genome browser, while open circle means the data were sequenced in present study. As for the three ENCODE region markers (AI, A5, and A6), these segments for baboon were retrieved from NCBI.

equal to the decay index for that node on the total evidence tree [25].

The results of the PBS analysis are presented in Table 4. The PBS values for 7 nodes across all the 10 markers and summed PBS scores for each marker are presented to evaluate the contribution of a given marker to the overall support of the simultaneous analysis tree. In order of total level of support, marker A5 shows the highest degree of support (56) for the simultaneous analysis tree, while A2 shows the lowest degree of support (16.5). For comparison, we also summarized the relative contribution of the support for the total autosomal markers (A1-A6) and X chromosomal makers (X1-X4), the concatenated dataset from the six autosomal markers shows a larger contribu- tion (258) to the whole tree than that from the four $\mathrm{X}$ chromosomal markers (175) (Table 4).

\section{Relative rate variation in primates}

To determine whether the rates of nucleotide substitutions for these 10 non-coding fragments varied between lineages on the phylogenetic tree, we performed relative rate tests. For example, to examine rate difference between apes and Old World monkeys, we used marmoset (a New World monkey) or the ring-tailed lemur (a strepsirrhine) as the outgroup (Table 5).

Pairs of lineages that exhibit significant rate differences are presented in Table 5. We observe that the Old World monkey lineage (as a group) is evolving significantly faster

Table 4: Partitioned Bremer support (PBS) for the simultaneous analysis parsimony tree.

\begin{tabular}{|c|c|c|c|c|c|c|c|c|}
\hline Marker & Node I & Node 2 & Node 3 & Node 4 & Node 5 & Node 6 & Node 7 & Total \\
\hline Al & 0 & 7.5 & 11.5 & 7 & 22 & 6 & 12 & 66 \\
\hline $\mathrm{A} 2$ & 0 & 5 & 0.5 & 3 & 3 & 5 & 4 & 20.5 \\
\hline A3 & 0 & 2.5 & 6 & 6 & 10 & 13 & 4 & 41.5 \\
\hline A4 & 0 & 3 & 12 & 3 & 12 & 11 & 6 & 47 \\
\hline A5 & 3 & 7 & 6 & 15 & 9 & 17 & 5 & 62 \\
\hline A6 & -2 & 6 & 2 & II & 15 & 21 & 4 & 57 \\
\hline$X I$ & 3 & 4 & 5.5 & 6 & 17 & 7 & 2 & 44.5 \\
\hline$\times 2$ & 2 & 4 & 4.5 & 7 & 15 & 15 & 4 & 51.5 \\
\hline$\times 3$ & 0 & I & 2.5 & 6 & 15 & 5 & I & 30.5 \\
\hline$\times 4$ & 2 & 3 & 5.5 & II & 23 & II & I & 56.5 \\
\hline Autosomes (Al-A6) & I & 31 & 38 & 45 & 71 & 73 & 35 & 294 \\
\hline X chr. $(X \mid-X 4)$ & 7 & 12 & 18 & 30 & 70 & 38 & 8 & 183 \\
\hline Concatenated & 8 & 43 & 56 & 75 & $|4|$ & III & 43 & 477 \\
\hline
\end{tabular}

Note. All marker rows show the PBS for each marker for the corresponding nodes shown in Figure 2. 
than the ape lineage. The lengths of Old World monkeyspecific branch are $30-50 \%$ longer than the ape-specific branch since their divergence, depending upon the choice of the outgroup (marmoset and lemur, respectively). This finding is in accord with the repeatedly observed 'hominoid/hominid-rate slowdown' phenomenon $[17,19,26$ 29].

Interestingly, we found that the macaque lineage is evolving significantly faster than the baboon lineage since the divergence of the two Old World monkey lineages. In the ten markers studied here (Table 5) the macaque lineage has accumulated almost twice as many nucleotide substitutions as has the baboon lineage. Indeed, analyses of ENCODE data have shown that the branch length leading to macaque is significantly longer than that leading to baboon [30]. Another recent study using over 8 million base pairs of aligned genomic sequences among several Old World monkeys also indicated significant rate difference between rhesus macaque and baboon [31]. Overall, New World monkeys evolve at a faster rate than the hominids (Table 5). We also discovered significant rate variation among New World monkeys. In particular, marmoset has a significantly faster substitution rate $(\mathrm{p}=0.015)$ than the spider monkey (Table 5).

These findings indicate that significant rate variation between lineages is a common feature of primate genome evolution. Based upon the known differences in life history traits [see Additional file 3], the observed rate differences are in general in agreement with the idea that species with larger body size, and likely longer generation time, tend to have slower molecular clocks. Baboons are larger and have longer lifespans than macaques, and spider monkey are larger and have longer lifespans than marmosets.

Taken together, these findings not only suggest a widespread influence of life history traits on molecular evolution of primates, but also provide a practical explanation on the fact that the degree of rate difference between specific primate groups can differ depending upon the actual lineages compared. For example, the hominid rate slowdown is more pronounced when rates are compared between human and macaque than between human and baboon (Table 5), which is in accord with the rate difference between macaque and baboon.

\section{Discussion}

Genomics is arguably one of the fastest evolving branches of modern science. Emerging new sequencing technologies enable analyses of large number of individuals from a species or to interrogate genetic diversity of a complex biological community. Yet, aside from the fields of microbial genomics and human population genomics, for most taxa on earth only a sparse amount of genomic resources are available.

Developing genome-scale markers from the majority of the diversity of life will have high payoff, facilitating ecological and evolutionary applications. Here we have illustrated that we can develop markers from specific genomic regions (such as non-coding, non-repetitive regions) utilizing genomic resources that are moderately divergent from target species. For example, studies estimate that the human/chimpanzee/macaque and the ring-tailed lemur has shared the last common ancestor up to 80 million years ago [32]. Here we have generated markers from potentially neutral genomic regions in ring-tailed lemurs using primers based upon human-chimpanzee-macaque genome alignments.

As far as we are aware, data from non-coding regions of strepsirrhines are rare, and markers developed in this study have a great potential to be used in evolutionary studies of this group. Importantly, we demonstrated that we could potentially achieve genome scale marker generation by sampling genomes across the tree of life with moderate divergence times.

Table 5: Relative rate test: Comparisons of evolutionary changes among several primate lineages.

\begin{tabular}{lcccccccc}
\hline Lineage I & Lineage 2 & Outgroup & KI & K2 & dK & sd_dK & P & Ratio \\
\hline OWM & HOM & Marmoset & 12.20 & 11.14 & 1.06 & 0.36 & $0.003^{* *}$ & 1.36 \\
OWM & HOM & Lemur & 23.48 & 22.20 & 1.28 & 0.42 & $0.002^{* *}$ & 1.48 \\
Macaque & Baboon & Marmoset & 12.48 & 12.08 & 0.40 & 0.18 & $0.029^{*}$ & 1.93 \\
Macaque & Baboon & Lemur & 23.86 & 23.42 & 0.44 & 0.21 & $0.039^{*}$ & 1.99 \\
Baboon & HOM & Marmoset & 12.00 & 11.14 & 0.86 & 0.37 & $0.019^{*}$ & 1.28 \\
Macaque & HOM & Marmoset & 12.42 & 11.15 & 1.26 & 0.38 & $0.000^{* * *}$ & 1.43 \\
Marmoset & Spider monkey & Lemur & 23.83 & 22.77 & 1.06 & 0.44 & $0.015^{*}$ & 1.48 \\
NWM & HOM & Lemur & 23.34 & 22.06 & 1.28 & 0.50 & $0.011^{*}$ & 1.24 \\
\end{tabular}

Notes. I. KI, number of substitutions per site that lineage I accumulated starting from the split with lineage 2; dK, the difference between $\mathrm{KI}$ and $\mathrm{K} 2$ numbers of substitutions; sd_dK, standard deviation for $\mathrm{dK} ; \mathrm{P}$, significance value for differences in substitution rates between lineage I and lineage 2; Ratio, the ratios of the branch length leading to the lineage I to that leading to the lineage 2 since the divergence of the two lineages. 2. $\mathrm{HOM}=$ hominoids, OWM $=$ Old World monkey, NWM = New World monkey.

3. *significant at 0.05 level; **significant at 0.01 level; ***significant at 0.00 I level. 
We chose Primates as the test group of organisms for this study because their phylogenetic relationships are well characterized [33]. We felt that if we could replicate the known primate phylogeny using the markers we designed then we would have proof of principle that this type of marker was useful for phylogenetic analysis. Toward that end this study was successful. Using a subset of new markers, we have performed phylogenetic analyses. The resulting phylogenies from single markers were generally in accord with the accepted phylogenetic relationship among different primate species. In particular, no marker supported incorrect phylogeny with statistical significance. The only node that required several markers to be resolved was the relationship among human, chimpanzee, and gorilla, a notorious phylogenetic example that had previously been shown to require a large amount of data to be resolved $[34,35]$. Given that there are several outstanding problems remaining in the field of primate phylogeny (e.g., [13-15,36]), putatively neutral markers such as developed in this study potentially will be useful toward resolving these issues.

Previous work focusing on identifying conserved, ultraconserved, or lineage specific elements for their potential functionality (e.g. [37,38]) have recognized the usefulness of utilizing regions located distantly from annotate genes as putatively 'neutral' standards to generate statistical 'background' for their analysis. Our approach, while seeking to identify regions of the genome that are not under functional constraint instead, have adopted such underlying logic and applied it to species whose genomic sequences are not yet available. It should be cautioned however that computational logics identifying putatively 'neutral' markers do not guarantee true neutrality: it remains as a prime challenge in modern genomics to experimentally establish neutrality or functionality of non-coding regions.

It has recently been demonstrated that protein coding regions are subject to frequent and widespread parallel evolution [6]; therefore we attempted to choose regions that are less likely to be subject to homoplastic effects that could result in misleading phylogenies. That our results support the well-known topology of Primates (e.g. [33]) is encouraging, because our understanding of primate phylogeny has taken over a century to get to the point is at today. There are still phylogenetic controversies (i.e. the hominoid trichotomy, the relationships among neotropical platyrrhines, and the phylogenetic placement of tarsiers) that still resist resolution besides decades of work.

Using the newly developed markers, we also observed substantial evolutionary rate variation among different primate lineages. We not only confirm the phenomenon of hominid rate slowdown $[17,19,26,29]$, but also uncov- ered several other intriguing patterns. In particular, we observe that since the divergence of rhesus macaque and anubis baboon (estimated to be approximately 6-8 millions of years ago, $[28,29])$, the macaque lineage has accumulated almost twice as many mutations as the baboon lineage. Substantial rate variation between Old World monkey lineages could have contributed to earlier controversy over the degree of hominid rate slowdown. When the macaque lineage is used to compare evolutionary rates of hominids and Old World monkeys, the degree of rate slowdown is much stronger (Table 5). We also observed a strong rate difference between marmoset and spider monkey, and to a lesser degree between tamarin and spider monkey. The marmoset lineage has accumulated almost $50 \%$ more mutations than the spider monkey lineage since the two lineages have split (Table 5). Thus, evolutionary rate variation is a common phenomenon in putatively neutral genomic regions [18].

The relative rate test shows that mutations have accumulated in the Old World monkey and New World monkey lineage at a rate significantly higher than in the hominids. We found no significant evolutionary rate difference between Old World monkeys and New World monkeys, in contrast to an earlier finding [28]. However, we should be careful in concluding the patterns of rate variation between groups based upon data from a few lineages. First there is the issue of regional rate variation within a genome. Also, as we have witnessed above, rates can vary dramatically between closely related lineages (such as macaque and baboon). The earlier analysis on rate variation between marmoset and Old World monkeys [28] was based upon a single, albeit long ( $59.8 \mathrm{kbps})$, orthologous region. The New World monkey species used in the previous analysis was marmoset, a fast evolving lineage. Thus the previous finding of significant rate variation between Old World monkeys and New World monkeys [28] may reflect the underlying molecular clock specific to that genomic regions and the sets of species. In this respect expanding the usage of non-coding, non-repetitive markers from many different genomic regions as developed here to other primate lineages will be highly useful to reconcile these conflicting results and to elucidate the patterns and causes of genomic neutral molecular clocks in primates.

The markers developed in this study [see Additional file 1] should thus be of great use for ecological and evolutionary applications in primates. In particular a subset of markers from closely related species can be used as 'local' markers to elucidate recent evolutionary events, while a few markers that can be amplified throughout the evolution of primates (such as the 10 markers analyzed here) could be used as 'global' markers to analyze underlying trends in primate evolution. 


\section{Conclusion}

Molecular markers from nuclear, putatively neutrally evolving genomic regions are extremely useful in ecological and evolutionary applications, yet hard to obtain from the majority of taxa. Here we describe a method for developing numerous nuclear markers from putatively neutral regions of primate genomes. First we demonstrate that by combining computational and experimental methods, we can generate a large number of putatively neutral markers from diverse primate genomes. Our PCR analyses show that we can amplify numerous markers from several wellseparated primate lineages. We chose a subset of ten markers among the newly developed markers and amplified them from five primate species. Notably, we were able to amplify and sequence a subset of these markers from a ring-tailed lemur, representing a lineage that diverged from the lineage leading species from which the primers were designed 80 million years ago. We show that these ten markers can reconstruct the phylogenetic relationships among the ten primate species with high confidence, and useful in analyses of evolutionary rate variation between lineages. In particular we uncovered substantial rate variation among lineages, both within and between different primate families. Thus, these markers can provide a snapshot of genomic divergences and are likely to be highly useful in diverse applications.

\section{Methods \\ Genome-scale mining of potential non-repetitive, non- coding amplicons}

Whole genome assemblies of human (H. sapiens), common chimpanzee ( $P$. troglodytes) and rhesus macaque $(M$. mulatta) (hg18, panTro2, rheMac2, respectively) were retrieved from the UCSC genome browser [39]. The human-chimpanzee-macaque alignments were obtained using blastz program [40]. Using the 'Ensembl' and 'Known' gene annotations provided by the UCSC genome browser, we identified non-coding regions, which include intergenic regions (defined as regions that are at least 1500 base pairs [bps] away from any known or ENSEMBL gene) and introns. We removed first introns and short (less than $250 \mathrm{bps}$ ) introns from further analyses, since these may be under selective constraint to preserve regulatory signals. We then extracted intergenic and intronic amplicons of length between 500 to 1500 bps, flanked by highly conserved 30-50 bp regions to be used as primer sites. Amplicons within 150 bps from an intronic start site or end site were also removed. We further filtered amplicons so that at least $80 \%$ of the sites are aligned between human-chimp-macaque and at least $85 \%$ of the aligned sites are from non-repetitive regions.

We then selected candidate primers, by either directly using the highly conserved flanking regions or automatically designing primers based upon the potential ampli- cons sequences, using the software fastPCR [41]. After designing primer pairs, we used either the BLAT [42] or BLAST [43] programs to map the primer pairs and the potential amplicons back to the human and macaque genome, to select only those that have a single hit in both human and macaque genome. This step removes potential spurious PCR products due to the homology to the primer sequences. Furthermore, we used BLAST program to map the single hit primers against the supercontig data of marmoset genome (downloaded from Washington University Genome Research Center). We chose primer pairs that satisfy the following criteria only: for each amplicon, the forward and the reverse primer should hit the same contig in marmoset genome, and the length of marmoset high scoring pair (HSP) should be at least 20 bps in length.

\section{Amplifying candidate amplicons from diverse primate genomes}

To demonstrate the usefulness of the markers developed by our computational approach, we constructed a ten-species data set for a subset of markers. This data set is comprised of experimentally gathered data from five species combined with computationally extracted data from additional five species. First, five species across diverse group of primates were chosen to test newly developed nonrepetitive and non-coding nuclear primer pairs. These are, gorilla (G. gorilla, a hominid), anubis baboon (P. anubis, an Old World monkey), black-handed spider monkey (A. geoffroyi, a New World monkey), white-lipped tamarin ( $S$. labiatus, a New World monkey), and ring-tailed lemur ( $L$. catta, a prosimian). DNA samples used in present study were purchased from Coriell (Camden, NJ) either as a primate phylogenetic panel (PRP00001) or individually (e.g., PR00036: anubis baboon).

To amplify the markers, approximately $5 \mathrm{ng}$ of genomic DNA was used as the template for a $10 \mathrm{ul} \mathrm{PCR} \mathrm{mixture.} \mathrm{We}$ used a "touchdown" PCR program with an initial annealing temperature at $60^{\circ} \mathrm{C}$ and temperature subsequently decreased by $1^{\circ} \mathrm{C}$ every cycle until the specified annealing temperature $50^{\circ} \mathrm{C}$ is reached. Each reaction was performed using the cycles of $30 \mathrm{sec}$ at $94^{\circ} \mathrm{C}, 30 \mathrm{sec}$ at 60 $50^{\circ} \mathrm{C}$, and $60 \mathrm{sec}$ at $72^{\circ} \mathrm{C}$, with an initial step of $3 \mathrm{~min}$ at $94^{\circ} \mathrm{C}$ and a final step of $4 \mathrm{~min}$ at $72^{\circ} \mathrm{C}$. Polymerase chain reaction products were purified using ExoSAP-IT (USB, Cleveland, USA) and then sequenced on both strands. The PCR primers were also used as sequencing primers. We performed gel purifications for the following three markers: A3 fragment from the baboon, A4 fragment from tamarin, and the $\mathrm{A} 1$ fragment from the lemur.

After sequencing, we used the BLAT program [42] to confirm that there is only one significant hit for each individual marker. These were combined with computationally 
extracted data from five other primate species. Specifically, homologous regions for the corresponding amplicons were retrieved from the UCSC genome browser from three hominids, including human (H. sapiens), chimpanzee ( $P$. troglodytes; we amplified the X1 experimentally), orangutan (P. pygmaeus abelii), and one Old World Monkey, rhesus macaque (M. mulatta; we amplified the A5 marker experimentally), and a New World Monkey, common marmoset (C. jacchus).

\section{Sequence analysis}

Initial sequence assembly was carried out using the Sequencher software [44]. Full alignments were produced with MAFFT using the L-INS-i MAFFT option [45]. All alignments were checked by eye, adjusted where necessary. Poorly aligned regions were removed using the Gblocks 0.91b program [46] with the setting of relaxed selection of blocks [47]. The final concatenated sequence alignment that was used for subsequent analyses contained 5592 aligned positions. Compositional stationarity was explored using the Chi-Square test in PAUP*4.0b10 [48].

Phylogenetic analyses were conducted by equally weighted maximum parsimony (MP) method, with gaps treated as missing data using PAUP, and by neighbor-joining (NJ) method, based on Kimura 2-parameter model using MEGA4.1Beta [20]. Maximum likelihood analyses were performed using both Garlio.96 [49] and PHYML3 [50] programs. The mixed-model analysis was implemented in MrBayes3.1.2 [51]. The best-fitting substitution models for the ML and Bayesian analyses were chosen by the Akaike information criterion (AIC) implemented in ModelTest3.7 [22] and MrModelTest2.3 [23]. Statistical support for the resulting phylogenies was assayed by conducting 1000 bootstrap pseudo-replicates for MP and NJ analyses as completed in PAUP and MEGA, and 100 bootstrap pseudo-replicates for ML analyses as completed in Garli and PHYML. Mixed-model Bayesian analysis also employed locus-specific models comparable to those chosen for ML analyses of individual genes. In MrBayes3.1.2, two independent sets of MCMC chains were run, each with three heated and one unheated chain for $2 \times 10^{6}$ generations. These were sampled every 100 generations. All analyses employed the default flat Dirichlet priors. The stationarity of each run was assessed by monitoring the convergence of the standard deviation of split frequencies and by graphing posterior probabilities of both runs against generations. The trees and parameter values from the pre-convergence "burn-in" phases of the runs were excluded.

The behavior of combining characters from different partitions was evaluated by examining the relative contribution, or utility, of data partitions to resolving relationships within the simultaneous analysis tree by calculating the Partitioned Bremer support (PBS) using TreeRot3 [24].

Significance of evolutionary rate variation among lineages were tested by relative rate test, using the program RRTree1.1 [52].

\section{Authors' contributions}

DW, ZP and SY conceived the study and ZP, NE, and SY designed the particular experiment for the current study. NE performed computational mining and ZP generated markers by experimental and computational methods. ZP, NE, DW, and SY wrote the manuscript. DW and SY provided materials and reagents. All authors read and approved the final manuscript.

\section{Additional material}

\section{Additional file 1}

Genomic non-coding, non-repetitive markers generated in present study. Primer ID, corresponding human chromosome, and primer sequences are listed for the 280 markers.

Click here for file

[http://www.biomedcentral.com/content/supplementary/14712164-10-247-S1.xls]

\section{Additional file 2}

Pairwise Kimura two-parameter distances among the 10 primate species. Pairwise Kimura two-parameter distances among the 10 primate species for each marker and for concatenated dataset are listed in the table. Click here for file

[http://www.biomedcentral.com/content/supplementary/14712164-10-247-S2.xls]

\section{Additional file 3}

Life history traits among anthropoid primate species in this study. Some important life history traits such as age of female sexual maturity, age at first birth, and lifespan are presented for the 9 ingroup species. Click here for file

[http://www.biomedcentral.com/content/supplementary/14712164-10-247-S3.doc]

\section{Acknowledgements}

This study has been supported by the Blanchard-Milliken Faculty Fellowship, the Alfred P. Sloan Foundation, and a grant from the National Science Foundation (BCS-075I48I) to SVY, and a NSF grant BCS-075I 508 to DEW. We thank Dr. Larissa Swedell for discussions on life history variations in Old World monkeys.

\section{References}

I. Vigilant L, Stoneking M, Harpending H, Hawkes K, Wilson AC: African populations and the evolution of human mitochondrial DNA. Science 1991, 253:1503-1507.

2. Zink RM, Barrowclough GF: Mitochondrial DNA under siege in avian phylogeography. Mol Ecol 2008, I 7:2 I07-2I 21 .

3. Waugh J: DNA barcoding in animal species: progress, potential and pitfalls. BioEssays 2007, 29(2): 188-197.

4. Lopez JV, Yuhki N, Masuda R, Modi W, O'Brien SJ: Numt, a recent transfer and tandem amplification of mitochondrial DNA to 
the nuclear genome of the domestic cat. J Mol Evol 1994, 39(2): 174-190.

5. Richly E, Leister D: NUMTs in sequenced eukaryotic genomes. Mol Biol Evol 2004, 2 I (6): I08I- 1084.

6. Rokas A, Carroll SB: Frequent and widespread parallel evolution of protein sequences. Mol Biol Evol 2008, 25:1943-1953.

7. Zhang J: Parallel adaptive origins of digestive RNases in Asian and African leaf monkeys. Nat Genet 2006, 38(77):819-823.

8. Salem A-H, Ray DA, Xing J, Callinan PA, Myers JS, Hedges DJ, Garber RK, Witherspoon DJ, Jorde LB, Batzer MA: Alu elements and hominid phylogenetics. Proc Natl Acad Sci USA 2003, I 00(22): |2787-I279|.

9. Chen FC, Li WH: Genomic divergences between humans and other hominoids and the effective population size of the common ancestor of humans and chimpanzees. Am J Hum Genet 200I, 68(2):444-456.

10. Ellegren H, Smith NG, Webster MT: Mutation rate variation in the mammalian genome. Current Opinion in Genetics \& Development 2003, 13(6):562-568.

II. Silva JC, Kondrashov AS: Patterns in spontaneous mutation revealed by human-baboon sequence comparison. Trends Genet 2002, 1 8:544-547.

12. Horvath JE, Willard HF: Primate comparative genomics: lemur biology and evolution. Trends in Genetics 2007, 23(4): I73- 182.

13. Meireles CM, Czelusniak J, Page SL, Wildman DE, Goodman M: Phylogenetic position of tarsiers within the order Primates: evidence from g-globin DNA sequences. In Tarsiers: Past, Present, and Future Edited by: Wright PC, Simons EL, Gursky S. New Brunswick, NJ: Rutgers University Press; 2003:145-160.

14. Opazo JC, Wildman DE, Prychitko T, Johnson RM, Goodman M: Phylogenetic relationships and divergence times among New World monkeys (Platyrrhini, Primates). Mol Phylogenet Evol 2006, 40:274-280.

15. Yoder AD: The phylogenetic position of the genus Tarsius: whose side are you on? In Tarsiers: Past, Present, and Future Edited by: Wright PC, Simons EL, Gursky S. New Brunswick, NJ: Rutgers University Press; 2003:161-175.

16. Kimura M: A simple method for estimating evolutionary rate of base substitution through comparative studies of nucleotide sequences. J Mol Evol 1980, 16: I I I-I20.

17. Kim S-H, Elango N, Warden CW, Vigoda E, Yi S: Heterogeneous genomic molecular clocks in primates. PLoS Genetics 2006, 2:el63.

18. Yi S: Understanding neutral genomic molecular clocks. Evol Biol 2007, 34:|44-I5|.

19. Yi S, Ellsworth DL, Li WH: Slow molecular clocks in Old World monkeys, apes, and humans. Mol Biol Evol 2002, I 9(I 2):219|-2198.

20. Tamura K, Dudley J, Nei M, Kumar S: MEGA4: Molecular Evolutionary Genetics Analysis (MEGA) software version 4.0. Mol Biol Evol 2007, 24(8): I596-I599.

21. Liu C, program Ncs, Zhao S, Bailey J, Sahinalp SC, Alkan C, Tuzun E, Green ED, Eichler EE: Analysis of primate genomic variation reveals a repeat-driven expansion of the human genome. Genome Research 2003, 13:358-368.

22. Posada D, Crandall KA: Modeltest: testing the model of DNA substitution. Bioinformatics 1998, 14:917-918.

23. Nylander JAA: MrModeltest v2. Program distributed by the author. Evolutionary Biology Centre, Uppsala University; 2004.

24. Sorenson MD, Franzosa EA: TreeRot, version 3. Boston University, Boston, MA; 2007.

25. Baker RH, DeSalle R: Multiple sources of character information and the phylogeny of Hawaiian drosophilids. Syst Biol 1997, 46(4):654-673.

26. Goodman M: Evolution of the immunologic species specificity of human serum proteins. Hum Biol 1962, 34:104-150.

27. Li W-H, Tanimura M: The molecular clock runs more slowly in man than in apes and monkeys. Nature 1987, 326:93-96.

28. Steiper ME, Young NM: Primate molecular divergence dates. Mol Phylogenet Evol 2006, 4I:384-394.

29. Steiper ME, Young NM, Sukrarna TY: Genomic data support the hominoid slowdown and an early Oligocene estimate for the hominoid-cercopithecoid divergence. Proc Nat Acad Sci USA 2004, I 01:17021-17026.

30. Margulies EH, Cooper GM, Asimenos G, Thomas DJ, Dewey CN, Siepel A, Birney E, Keefe D, Schwartz AS, Hou M, et al: Analyses of deep mammalian sequence alignments and constraint predictions for $\mathbf{I \%}$ of the human genome. Genome Res 2007, I7(6):760-774.

3I. Elango N, Lee J, Peng Z, Loh Y-HE, Yi SV: Evolutionary rate variation in Old World monkeys. Biology Letters 2009, 5(3):405-408.

32. Yoder AD, Yang Z: Divergence dates for Malagasy lemurs estimated from multiple gene loci: geological and evolutionary context. Mol Ecol 2004, I3:757-773.

33. Goodman M, Grossman LI, Wildman DE: Moving primate genomics beyod the chimpanzee genome. Trends Genet 2005, 2I(9):5II-5I7.

34. Elango N, Thomas JW, Program NCS, Yi S: Variable molecular clocks in hominoids. Proc Nat Acad Sci USA 2006, I03:1370-I 375.

35. Wildman DE, Uddin M, Liu G, Grossman LI, Goodman M: Implications of natural selection in shaping $\mathbf{9 9 . 4 \%}$ nonsynonymous DNA identity between humans and chimpanzees: enlarging genus Homo. Proc Natl Acad Sci USA 2003, 100:7|8I-7/88.

36. Horvath JE, Weisrock DW, Embry SL, Fioretino I, Balhoff JP, Kappeler P, Wray GA, Willard HF, Yoder AD: Development and application of a phylogenomic toolkit: resolving the evolutionary history of Madagascar's lemurs. Genome Res 2008, 1 8:489-499.

37. Siepel A, Bejerano G, Pedersen JS, Hinrichs A, Hou M, Rosenbloom K, Clawson H, Spieth J, Hillier LW, Richards S, et al.: Evolutionary conserved elements in vertebrate, insect, worm, and yeast genomes. Genome Res 2005, I 5: 1034-1050.

38. Visel A, Prabhakar S, Akiyama JA, Shoukry M, Lewis KD, Holt A, Plajzer-Frick I, Afzal V, Rubin EM, Pennacchio LA: Ulltraconservation identifies a small subset of extremely constrained developmental enhancers. Nat Genet 2008, 40(2): I58-160.

39. Kent WJ, Sugnet CW, Furey TS, Roskin KM, Pringle TH, Zahler AM, Haussler D: The human genome browser at UCSC. Genome Res 2002, I 2(6):996-1006.

40. Schwartz S, Kent WJ, Smit A, Zhang Z, Baertsch R, Hardison RC, Haussler D, Miller W: Human-mouse alignments with BLASTZ. Genome Res 2003, I3(I):103-107.

4I. Kalendar R: FastPCR software for PCR primer and probe design and repeat search. 2009 [http://www.biocenter.helsinki.fi/ bi/programs/fastpcr.htm].

42. Kent WJ: BLAT - the BLAST-like alignment tool. Genome Res 2002, 12:656-664.

43. Altschul DA, Gish W, Miller W, Myers EW, Lipman DJ: Basic local alignment search tool. J Mol Biol I990, 21 5:403-4I0.

44. GeneCodes: Sequencher 4.0. [http://www.genecodes.com/]

45. Katoh K, Toh H: Improved accuracy of multiple ncRNA alignment by incorporating structural information into a MAFFT. based framework. BMC Bioinformatics 2008, 9:2I 2 .

46. Castresana J: Selection of conserved blocks from multiple alignments for their use in phylogenetic analysis. Mol Biol Evol 2000, I 7(4):540-552.

47. Talavera G, Castresana J: Improvement of phylogenies after removing divergent and ambiguously aligned blocks from protein sequence alignments. Syst Biol 2007, 56(4):564-577.

48. Swofford D: PAUP*: Phylogenetic analysis using Parsimony* (and other methods), 4.0bl0. Sunderland, MA: Sinauer Associates; 2002.

49. Zwickl DJ: Genetic algorithm approaches for the phylogenetic analysis of large biological sequence datasets under the maximum likelihood criterion. University of Texas at Austin; 2006.

50. Guindon S, Gascuel O: A simple, fast, and accurate algorithm to estimate large phylogenies by maximum likelihood. Syst Biol 2003, 52(5):696-704.

5I. Ronquist F, Huelsenbeck JP: MrBayes 3: Bayesian phylogenetic inference under mixed models. Bioinformatics 2003, 19:1572-1574.

52. Robinson-Rechavi M, Huchon D: RRTree: relative-rate tests between groups of sequences on a phylogenetic tree. Bioinformatics 2000, 16(3):296-297. 